\title{
Tilting Theory: A Gift of Representation Theory to Mathematics
}

\section{Gabriella D'Este}

Dedicated to the memory of Sheila Brenner and Michael C. R. Butler

1 $\mathrm{n}$ the introduction to his book Tame Algebras and Integral Quadratic Forms, Claus Michael Ringel remarks that "some general ideas, which have influenced the results and the methods presented here, are not available in official publications, or not even written up." This resonates with my personal experiences with both Places and People, to use the terminology of FDLIST [20]. This article is the written version of conversations I've had through the years with young colleagues on the unofficial history of tilting theory, its general ideas, unexpected facts, and open problems.

The origin of tilting theory in Italy is one of the best examples of the complexity of general ideas (and their zigzag journeys). Menini's paper [27] describes the role played by Adalberto Orsatti and his Algebra Team in Padova, and it contains an account of important public events and official publications, as well as information on private conversations, classical letters, and unexpected connections between distant places and people. The first paper by Italian authors on tilting theory is by Menini and Orsatti [28]. In the introduction of [28], the authors thank Masahisa Sato, Enrico Gregorio, and me. I regret that I have never expressed officially-in a paper-my thanks (and surprise) for this unexpected reference, as I should have done long ago. One aim of this article is to fill this gap, at least partially.

In the introduction to the Handbook of Tilting Theory [2], the authors state the following:

"Tilting theory arises as a universal method for constructing equivalences between categories. Originally introduced in the context of module categories over finite-dimensional algebras, tilting theory is now considered an essential tool in the study of many areas of mathematics, including finite and algebraic group theory, commutative and noncommutative algebraic geometry, and algebraic topology."

Perhaps it is for this reason that the referee of [15] wrote "tilting theory is the gift of algebra representation theory to mathematics." This remark inspired the title of this article.

"Tilting" has an immediate visual connotation, and readers unfamiliar with the history of the field might wonder. I refer to the Brenner \& Butler quote at the top of the Wikipedia article on tilting theory (https://en.wikipe dia.org/wiki/Tilting_theory) for the origin of the term. People care about tilting modules, because it is often useful to compare the categories of modules over $A$ and that of 
modules over the endomorphism ring $B$ of a tilting $A$ module $T$, which sometimes is a better-behaved ring.

It is likely that only a reader who has at least some passing acquaintance with homological algebra and the representation theory of finite-dimensional algebras will best appreciate the nuances of the mathematics described here. However, I hope that the story I tell here will offer some insight into this beautiful subject for most readers. There will be a lot of pictures, as making pictures has taught me much about tilting theory. Here are the lessons I learned by making pictures of tilting-type objects:

(a) "Simple" and combinatorial objects may have unexpected concealed topological properties (see "Do Finite-Dimensional Bimodules Have a Topology?").

(b) "Nonsimple" objects may have unexpected concealed discrete properties (See "Do Infinite-Dimensional Modules Need No Topological Tools?").

In the following, $K$ denotes an algebraically closed field, and we assume that all vector spaces and algebras are defined over $K$.

\section{A Letter from Japan and My First Homework on Tilting Theory}

Menini [27, page 11] describes Sato's contribution to her joint paper with Orsatti [28]:

"I would like to recall here that it was Masahisa Sato that pointed out to us that tilting modules might provide examples ... In fact Orsatti explained this problem to Sato during a NATO meeting held in Antwerpen (Belgium) in the period July 20-29, 1987. After some time Sato wrote to Orsatti showing an example of a tilting module."

I remember very well what happened next. Orsatti told me to make a copy of the letter received from Japan. My homework was to look at Happel and Ringel's example of a tilting module described in the last page of [21] and to give a talk about it. So my unique and small contribution to [28] was just a talk. And hence my surprise for those unexpected thanks.

Now I realize that I should have thanked Menini and Orsatti for the opportunity. While preparing for the talk I was pleasantly surprised to observe directly that Auslander-Reiten quivers can help one guess and see possible equivalences before constructing a proof of their existence. Indeed tilting equivalences and cotilting dualities also have a combinatorial nature, inherited and suggested by that of quivers and modules.

I was also able to discover the magic power of Auslander's formula, which reduces the computation of $\mathrm{Ext}^{1}$-groups to that of Hom-groups. (For details, see [3, Proposition 4.6 and Corollary 4.7] or [32, conditions (5) and (6), pages 75-76]). Consequently we may verify the vanishing of certain $\mathrm{Ext}^{1}$ groups by simply looking at the Auslander-Reiten quiver. Without this formula, I would be unable to check that Happel and Ringel's example is Ext ${ }^{1}$-self-orthogonal.

Picture 1 below (depicting a very combinatorial object with several symmetries) illustrates the shape of HappelRingel's bimodule $T={ }_{A} T_{B}$, where $B=\operatorname{End}_{A} T$. It is good to consider the whole bimodule ${ }_{A} T_{B}$, and not only the underlying module ${ }_{A} T$, in order to visualize equivalences and dualities.

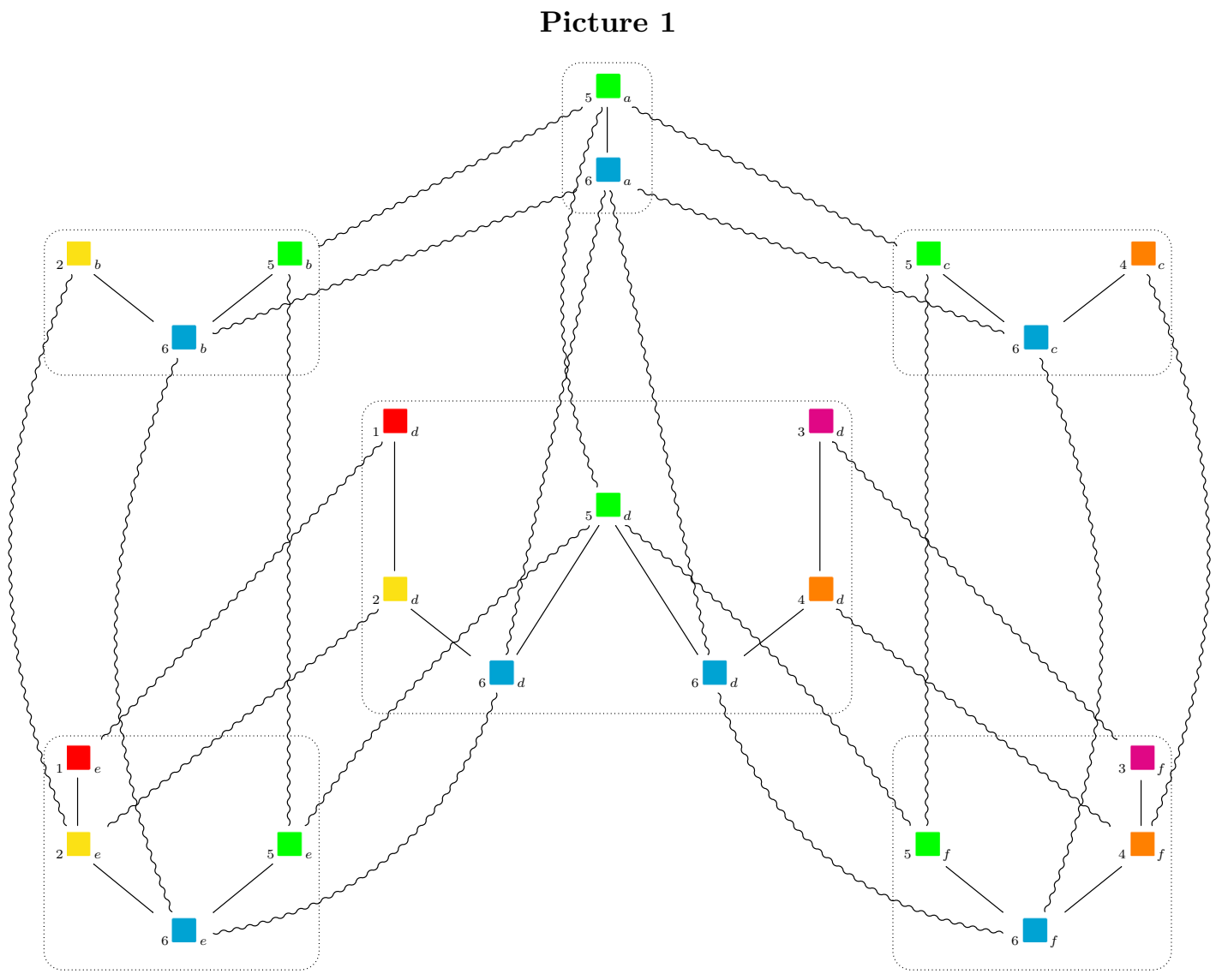


When drawing figures representing bimodules, we adopt the following conventions:

- Every square indicates an element $v$ of a fixed basis of the underlying vector space of $T$.

- The index $x$ on the left (resp. $y$ on the right) of a small square corresponding to the vector $v$ indicates that $e_{x} v=v=v e_{y}$, where $e_{i}$ is the path of length zero around the vertex $i$.

- Following Ringel's suggestion during my stay in Bielefeld, the small squares have a special position, so that they also describe in an obvious way the composition factors of the same module. (See, for instance, [29, 30] and [31, page 126] for descriptions and/or pictures of complicated modules.)

- We recall that the bimodules associated to "valued" arrows in [19] and [18] gave me the idea of adding two indices (on the left and on the right of the small squares). In this way we can see the action of left or right multiplication by the primitive idempotents, corresponding to vertices of some quiver.

- Straight lines describe left modules and wavy lines describe right modules. Recall that a representation of a quiver consists of vector spaces (one for each vertex) and of linear maps (one for each arrow) eventually satisfying suitable relations.

According to [21], the underlying vector space of $T$ has dimension 23, whereas $A$ is the algebra given by the Dynkin quiver $E_{6}$ with "subspace orientation," that is of the form

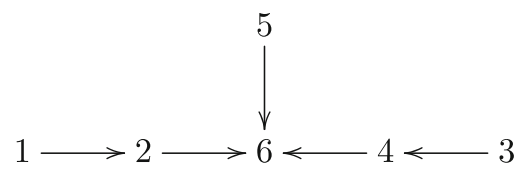

On the other hand, the algebra $B=\operatorname{End}_{A} T$ is isomorphic to the algebra given by the fully commutative quiver

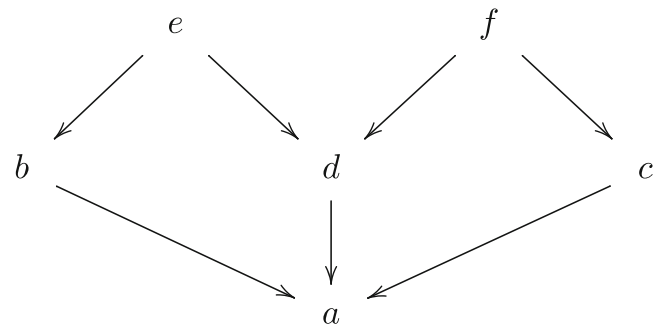

Now Picture 1 is not as old as my homework. I used similar pictures to visualize rather small bimodules for the first time, and more or less by chance. Of course, for big, complicated bimodules, it would be better to replace a 2dimensional "global" visualization (of the main properties of the left and right underlying modules) by a 3-dimensional one, without or with few self-intersections. However, even flat pictures such as Picture 1 are powerful enough to give for free a lot of indirect information in a compact way.

For example, Picture 1 tells us how the tilting equivalence represented by the functor $\operatorname{Hom}\left({ }_{A} T_{B},-\right)$ (between the modules generated by the left $A$-module $T$ and the modules cogenerated by the left $B$-module $D\left(T_{B}\right)=\operatorname{Hom}_{K}\left(T_{B}, K\right)$ ) acts on some indecomposable modules. For instance, the indecomposable summands of ${ }_{A} T$ are sent via this functor to the following indecomposable summands of ${ }_{B} B$ :

$$
\begin{aligned}
& \begin{array}{c}
5 \\
6
\end{array} \mapsto a, \quad \begin{array}{c}
25 \\
6
\end{array} \mapsto \begin{array}{c}
b \\
a
\end{array}, \quad \begin{array}{c}
54 \\
6
\end{array} \mapsto \begin{array}{c}
c \\
a
\end{array},
\end{aligned}
$$

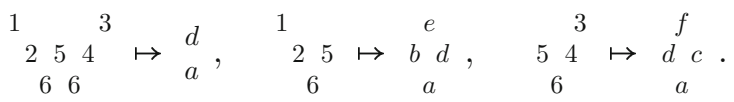

On the other hand, Picture 1 also describes how the cotilting duality induced by $\operatorname{Hom}\left(-,{ }_{A} T_{B}\right)$ (between the modules cogenerated by left $A$-module $T$ and the modules cogenerated by the right $B$-module $T$ ) acts on some indecomposable modules. For instance, the indecomposable summands of ${ }_{A} T$ are sent via this functor to the following indecomposable summands of $B_{B}$ :

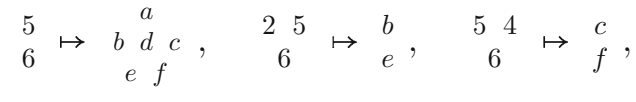

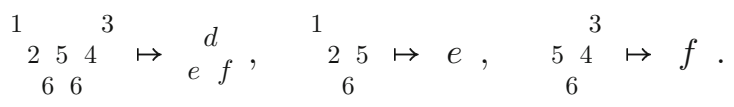

\section{What Happened Next: Talk with Big Matrices, More or Less Abstract Cancellations...}

The analysis of Happel-Ringel's bimodule was both my first homework on tilting theory, and the subject of my first talk on this subject. This was not my first talk in Padova containing some quivers. However, all the quivers I used earlier were much smaller and so much easier to describe without pictures. When I tried to do the same with Happel-Ringel's example, I realized that even basic techniques of representation theory of finite-dimensional algebras can make otherwise invisible things become visible.

For instance, to give a definition of $A$ and $B$ without quivers, I distributed some pages with the largest matrices I ever used, namely $23 \times 23$ matrices, which I did not want to draw at the blackboard. After some time I noticed that I could use more reasonable matrices to describe the $K$-linear maps from $T$ to $T$, corresponding to multiplications by elements of $A$ or $B$. Indeed, $T$ has the strong property that the groups of all morphisms between two indecomposable summands of ${ }_{A} T$ and $T_{B}$, respectively, are $K$-vector spaces of dimension at most one. Hence, after cancellation of many "inessential" rows and columns, one can see that $A$ 
and $B$ are isomorphic to subalgebras of the algebra of all $6 \times 6$ matrices of the form:

$$
\begin{gathered}
\left(\begin{array}{cccccc}
K & 0 & 0 & 0 & 0 & 0 \\
K & K & 0 & 0 & 0 & 0 \\
0 & 0 & K & 0 & 0 & 0 \\
0 & 0 & K & K & 0 & 0 \\
0 & 0 & 0 & 0 & K & 0 \\
K & K & K & K & K & K
\end{array}\right) \\
\text { and } \\
\left(\begin{array}{cccccccc}
K & K & K & K & K & K \\
0 & K & 0 & 0 & K & 0 \\
0 & 0 & K & 0 & 0 & K \\
0 & 0 & 0 & K & K & K \\
0 & 0 & 0 & 0 & K & 0 \\
0 & 0 & 0 & 0 & 0 & K
\end{array}\right) .
\end{gathered}
$$

Endomorphism rings of abelian groups were the subject of my master's thesis, "Abelian Groups Whose Endomorphism Ring is Locally Compact in the Finite Topology," written under the direction of Adalberto Orsatti. Moreover, some of my first papers dealt with endomorphism rings. However, without using quivers, this previous abstract experience on (usually large) endomorphism rings wouldn't have been useful to verify that $B=\operatorname{End}_{A} T$ actually had the indicated form.

The cancellation of rows and columns was only the first of many other (more abstract) cancellations made in the sequel, concerning modules and complexes (see the sections entitled "Cancellation of Summands" and "An Example of Cancellations"). For more on the theoretical importance of cancellations in tilting theory, I refer the reader to Ringel's lecture [33] on the occasion of the 20th anniversary of the Department of Mathematics of the University of Padova [see the section "Fully Documented Lectures" in Ringel's home page]. Note that the suggestive title of this lecture is "Tilting Theory: the Art of Losing Modules."

\section{Short or Long Definitions With or Without Classes of Modules}

Definitions Long and Short. The most compact and elegant definitions of tilting modules and of partial tilting modules, natural generalizations of tilting modules, consist of precisely one rather short property. For instance, any "classical" tilting (resp. cotilting) $R$-module $M$ (hence also Happel-Ringel's module $T$ ) has the property that the class of all modules generated (respectively, cogenerated) by $M$ coincides with the kernel of $\operatorname{Ext}_{R}^{1}(M,-)$ (respectively, $\operatorname{Ext}_{R}^{1}(-, M)$ ). This global property is equivalent to a much longer characterization capturing three discrete properties of two modules, namely of $M$ and of the regular module $R$ (respectively, an injective cogenerator $Q$ ). For me it was always easier to deal with more than one (but finitely many) elementary properties instead of dealing with just one property on classes of modules.

Let us begin with one such longer definition, for the classical case. ${ }^{1}$ We say that a module $M$ is a classical tilting or cotilting module (more precisely, a 1-tilting or 1-cotilting module), respectively, if the following conditions hold:

- The projective (respectively, injective) dimension of $M$ is at most 1 .

- $\operatorname{Ext}_{R}^{1}(M, \oplus M)=0 \quad$ (respectively, $\left.\operatorname{Ext}_{R}^{1}(\Pi M, M)=0\right)$, where $\oplus M$ (respectively, $\Pi M$ ) is any direct sum (respectively product) of copies of $M$.

- There is a short exact sequence of the form

$$
0 \longrightarrow R \longrightarrow M^{\prime} \longrightarrow M^{\prime \prime} \longrightarrow 0
$$

(respectively, $0 \longrightarrow M^{\prime} \longrightarrow M^{\prime \prime} \longrightarrow Q \longrightarrow 0$ ), where $M^{\prime}$ and $M^{\prime \prime}$ are direct summands of direct sums (respectively, products) of copies of $M$.

For finite-dimensional modules over finite-dimensional algebras, the setting studied by Brenner and Butler [5], the third condition may be replaced [32, page 167] by a numerical condition:

The number of isomorphism classes of indecomposable summands of $M$ is equal to the rank of the Grothendieck group of $R$.

Next consider two short definitions (for the general case). Given an $R$-module $M$ and a natural number $n>0$, we denote by $\operatorname{Gen}_{n}(M)$ the class of all modules $X$ such that there is an exact sequence of the form

$$
M(1) \longrightarrow \ldots \longrightarrow M(n) \longrightarrow X \longrightarrow 0,
$$

where the $M(i)$ 's are direct summands of direct sums of copies of $M$. Following [4], we say that $M$ is a tilting (respectively partial tilting) module of projective dimension at most $n$ if $\operatorname{Gen}_{n}(M)$ is equal to (respectively, is contained in) the orthogonal class

$$
M^{\perp}=\bigcap_{i>0} \operatorname{Ker} \operatorname{Ext}_{R}^{i}(M,-) .
$$

As in the classical case, the long definition of such $M$ consists of two or three conditions on just two modules, namely on $M$ and a very special projective or injective module.

Here is one of the reasons that it may be difficult to check equalities or inclusions of classes of modules that play a key role in the characterization of "nonclassical" tilting and cotilting-type modules $M$. Even in dealing with algebras of finite representation type, in the "nonclassical" case, these modules $M$ have the property that one of these two classes of modules (namely an orthogonal class) is closed under direct summands, whereas the other does not necessarily have this closure property. To see this, it

\footnotetext{
${ }^{1}$ See [4, Proposition 3.6 and Lemma 3.12] for the beautiful technical condition (on the relationship between two classes of modules), which characterizes "nonclassical" tilting or cotilting modules and their generalizations.
} 
suffices to consider the following example. Let $R$ be the algebra given by the quiver

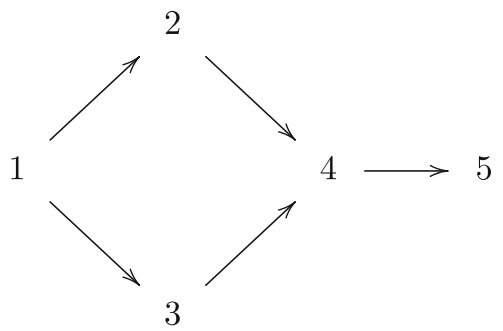

such that the composition of any two arrows is zero. Then we deduce from [14, Example B] that the injective module $T={ }_{5}^{4} \oplus{ }^{2}{ }^{3} \oplus{ }_{3}^{1} \oplus{ }_{2}^{1}$ is a partial tilting module (of projective dimension 3) such that $\operatorname{Gen}_{3}(T)$ contains the module $1 \oplus 1$ but not its summand 1 . Moreover both $\operatorname{Gen}_{3}(T)$ and the class $\operatorname{Add}(T)$, formed by all injective $R$ modules without simple summands, have the same indecomposable modules, namely the 4 indecomposable summands of $T$. This means that we cannot determine the class $\operatorname{Gen}_{3}(T)$ by just looking at the Auslander-Reiten quiver. In other words, the operation of making direct sums (the "only really well-understood construction" [36, page 476]) is not enough to investigate an important class of modules generated by a maximal direct summand of a tilting module of projective dimension 3. Note that $T$ is an example of a faithful module that is not tilting.

Cancellation of Summands. Let us next consider the cancellation of an injective nonprojective summand (to obtain a "large" partial tilting module). We obtain the faithful module $T$ constructed above from the minimal injective cogenerator $D\left(R_{R}\right)=\operatorname{Hom}_{K}\left(R_{R}, K\right)$ by means of cancellation of its injective summand 1 (of projective dimension 3). On the other hand, $\operatorname{Ext}_{R}^{2}(T, 5) \neq 0$ and 5 is the unique indecomposable module $X$ such that $\operatorname{Hom}_{R}(T, X)=0$. Consequently, we have

$$
\text { (*) } \quad \operatorname{Ker} \operatorname{Hom}_{R}(T,-) \bigcap T^{\perp}=0 .
$$

In other words, $T$ is a large partial tilting module in the sense of [13].

Large partial tilting modules are partial tilting modules satisfying $(*)$. Any tilting module is a large partial tilting module [4, page 371]. Any finitely-generated large partial tilting module of projective dimension at most 1 is a tilting module [6, Theorem 1]. Now Property (*) and [13, Lemma 11.2] imply that any large partial tilting module over a semiperfect ring, say again $T$, is sincere [24], that is, it has the property that $\operatorname{Hom}(P, T) \neq 0$ for every projective module $P \neq 0$. Consequently, a module $T$ of finite length is sincere if every simple module is a composition factor of $T[3,32]$.

Now let us look at the cancellation of a projectiveinjective summand of a tilting module with minimal orthogonal class (to obtain a "large" partial tilting module). Let $R$ be a finite-dimensional algebra or, more generally, a noetherian and semiperfect ring such that every indecomposable injective module has a simple socle. Let $M$ be an injective tilting $R$-module (of projective dimension $>1$ ) such that the orthogonal class $M^{\perp}=$

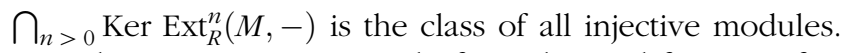
Let $T$ be a sincere summand of $M$, obtained from $M$ after cancellation of a projective summand $P$. Then we deduce from [13, Theorem 11.4] that $T$ is a large partial tilting module. [We will soon give a "minimal" example of this result, where $T$ is

(1) the unique indecomposable injective module that is not projective;

(2) a uniserial module such that every simple module has multiplicity one as a composition factor of $T$, that is a sincere module of minimal dimension.

Cancellations have both theoretical and practical importance in tilting theory. It turns out that various types of cancellations can help us deal with more abstract partial tilting objects. For instance, this often happens with partial tilting complexes, say $T^{\circ}$, in the sense of Rickard [34] with the following property:

(a) $T^{\circ}$ is the projective resolution of a large partial tilting module $T$, which is not a tilting module.

By [34], this hypothesis guarantees the existence of a nonzero right bounded complex (of projective modules) $X^{\circ}$ with the following property:

(b) $X^{\circ}$ is not the projective resolution of a module and every morphism from $T^{\circ}$ to any shift of $X^{\circ}$ is homotopic to zero.

The examples constructed in [15, 16, 17] suggest that there is no canonical way to obtain $X^{\circ}$ from $T^{\circ}$. Moreover, the same holds by confining ourselves to complexes $T^{\circ}$ and $X^{\circ}$ satisfying $(a)$ and (b), respectively, and with the following additional "very combinatorial" property (in the words of [35]):

(c) Any nonzero component of the indecomposable summands of $T^{\circ}$ and $X^{\circ}$ is an indecomposable module.

Indeed, up to shift, the choices of the indecomposable complexes $X^{\circ}$ satisfying both $(b)$ and $(c)$ may be quite different. For instance, there may be either zero [15, Example C (iii) and (iv)], or one [17, Remark after Example 1], or infinitely but countably many [17, Remark after Example 3], or uncountably many [17, Example 4] choices. The following example shows that by deleting some components of an indecomposable complex $T^{\circ}$, with properties ( $a$ ) and (c), we may obtain all the shortest complexes $X^{\circ}$ with properties $(b)$ and $(c)$, that is, the "elementary" complexes in the sense of [35] of the form

(d) $0 \longrightarrow P \longrightarrow Q \longrightarrow 0$ with $P$ and $Q$ indecomposable projective modules.

An Example of Cancellations. For every even integer $m>2$, there is a uniserial nonfaithful injective module $T$ (of projective dimension $m$ ) such that we obtain all the indecomposable complexes $X^{\circ}$ with the above properties (b) and ( $d$ ) by means of various types of cancellations of some components of $T^{\circ}$, that is, left cancellations, right cancellations, and, sometimes, central cancellations also. 
Indeed, let $A$ be the Nakayama algebra [26] given by the quiver

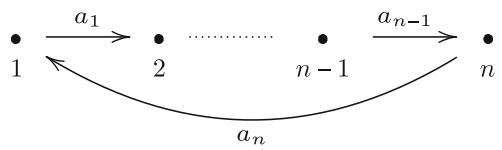

with relation $a_{n} \cdots a_{1}=0$, where $2 n=m+2$. Next, let $T$ denote the injective module of the form

$$
\begin{gathered}
2 \\
3 \\
\vdots \\
n \\
1
\end{gathered}
$$

We obtain $T$ from the minimal injective cogenerator $D\left(A_{A}\right)=\operatorname{Hom}_{K}\left(A_{A}, K\right)$ after cancellation of its $n-1$ indecomposable projective summands. Thus we deduce from the "Cancellation of Summands" section (or from [13, Example 11.6]) that $T^{\perp}$ is the class of all injective modules and $T$ is a large partial tilting module of projective dimension $m$. (The letter $n$ in condition (c) of [13, Example 11.6] is a misprint; it should be $m$.) Moreover the projective resolution $T^{\circ}$ of $T$ satisfies $(a)$ and $(c)$, and the complexes $X^{\circ}$ satisfying $(b)$ and $(d)$ are of the form

$$
0 \longrightarrow I(i) \longrightarrow I(j) \longrightarrow 0,
$$

where $I(*)$ denotes the indecomposable injective module corresponding to the vertex $*$ and $i>j>1$ [16, Proposition 1]. Hence they are exactly the complexes with two nonisomorphic injective components different from zero, obtained from $T^{\circ}$ after suitable cancellations.

\section{Do Finite-Dimensional Bimodules Have a Topology?}

Visualizing bimodules as in the "A Letter from Japan and My First Homework on Tilting Theory" section, I could first "see" and then prove that even rather small bimodules behave badly with respect to some quite natural constructions such as embeddings into bimodules with an underlying left (or right) injective module. Indeed, according to certain results on the socle of $E(C) / C$ [25, Lemma 2.2] and on the modules cogenerated by $E(C) / C$ [25, Propositions 1.7 and 2.1 and Theorem 1.17], it is natural to measure the gap between a cotilting module $C$ and its injective envelope $E(C)$, at least for modules that are finite-dimensional vector spaces. However, in this special situation where the discrete topology seems to be the most natural topology, two radically different situations show up.

Bad Case. It is not always possible to embed a finite-dimensional cotilting bimodule $C$ in another bimodule $D$ with the property that $D$, as a left (respectively, right) module is the injective envelope of $C$ [12, Example B (c), (d)]. Moreover, no left-right symmetry exists, because only one of the constructions may be possible [12, Example A (c), (d)].

Good Case. When such an embedding exists, the structure of $D$ as a right (respectively, left) module seems to be the most obvious one. Multiplications on the opposite side, that is, right (respectively, left) multiplications, are described by nice matrices with many entries equal to zero; they seem to be "continuous" extensions of their restriction to $C$. However, as the following toy example shows, the property of being an indecomposable bimodule is neither hereditary nor left-right symmetric.

Toy Example of a Finite-Dimensional Cotilting Bimodule [12, Example D] . Let $S$ (respectively, $R$ ) be the algebra given by the quiver

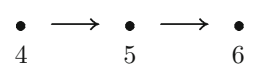

(respectively, $\dot{ }_{2} \longrightarrow \dot{i}_{1} \longleftarrow \dot{ }_{3}$ ), and let ${ }_{S} C_{R}$ be an indecomposable cotilting bimodule with

$$
\begin{aligned}
& { }_{S} C=\begin{array}{l}
4 \\
5
\end{array}{ }_{6}^{5} \oplus 5 \\
& \text { (respectively, } \left.C_{R}={ }_{2}^{1}{ }_{3}^{1} \oplus{ }_{2}^{1} \oplus 2\right)
\end{aligned}
$$

of the form

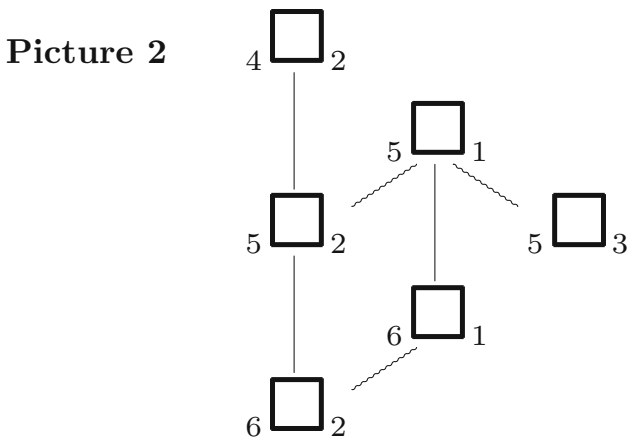

Then $C$ has codimension 2 in its left (respectively, right) injective envelope

$$
\begin{aligned}
& E(C)=\begin{array}{l}
4 \\
5 \\
6
\end{array} \oplus \begin{array}{l}
5 \\
5
\end{array} \oplus \begin{array}{l}
4 \\
5
\end{array} \\
& \text { (respectively, } \left.E(C)=\frac{1}{2} \oplus \begin{array}{l}
1 \\
2
\end{array} \oplus_{2}^{1} \oplus \begin{array}{l}
1 \\
3
\end{array}\right) \text {. }
\end{aligned}
$$

Moreover $E(C)$ is the support of an indecomposable (respectively, a decomposable) bimodule $D$, containing $C$ as a bimodule, of the form

Picture 3

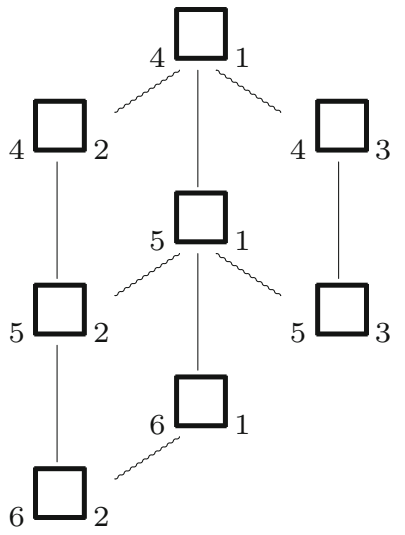




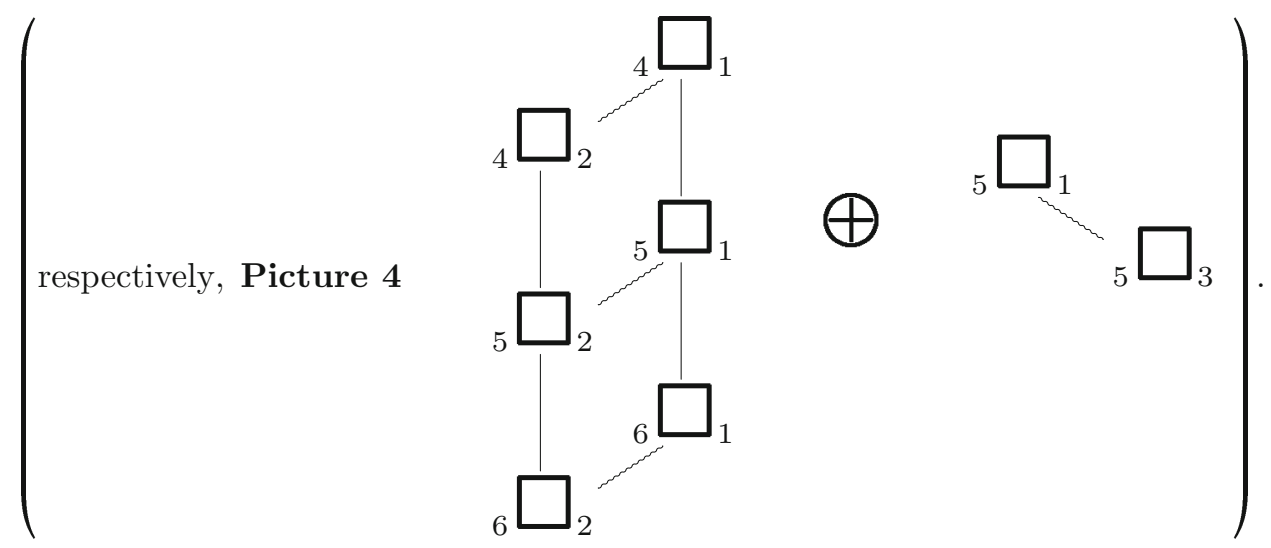

Remarks on the Action of Primitive Idempotents and Nilpotent Elements in the Good Case. In all the examples constructed in [12], where ${ }_{S} C_{R}$ is a cotilting bimodule such that $E\left({ }_{S} C\right)$ admits a structure of $S-R$ bimodule, containing the cotilting bimodule ${ }_{S} C_{R}$, we see that the $\operatorname{ring} R$ is hereditary. Furthermore, if $X$ is an indecomposable summand of ${ }_{S} C$ and $e$ is a primitive idempotent of $R$ such that $x e=x$ for every element $x \in X$, then we also have $x^{\prime} e=x^{\prime}$ for every element $x^{\prime} \in E\left({ }_{S} X\right)$. Finally the nilpotent elements of $S$ and $R$ corresponding to arrows act on the elements of $E(C) \backslash C$ in the easiest possible way, by a kind of shift. Indeed, if $s \in S, r \in R$ and $u, v, w$ (resp. $u, w, w, x)$ are linearly independent elements of $C$ (resp. of $E(C)$ ) such that $x \in E(C) \backslash C, s x=v, s w=u$, vr $=u$, then we have $x r=w$, as illustrated in Picture 5:

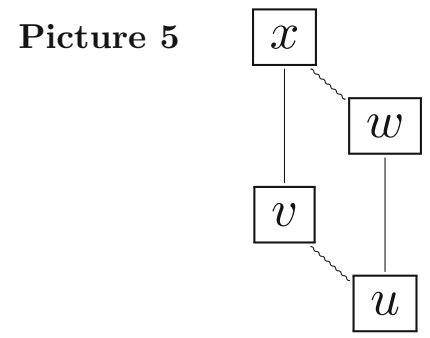

Roughly speaking, the above picture says that the vectors $x$ and $v$ have similar neighborhoods, and so the whole bimodule looks like a topological object.

Remarks on the Action of New Concealed Rings in the Bad Case. In all the examples constructed in [12], where ${ }_{S} C_{R}$ is a cotilting bimodule such that $E\left({ }_{S} C\right)$ does not admit a structure of $S-R$ bimodule containing the cotilting bimodule ${ }_{S} C_{R}$, we can show that

(i) The ring $R$ is not hereditary.

(ii) We can find a ring $R^{*}$, a ring epimorphism $F: R^{*} \longrightarrow R$, and a bimodule ${ }_{S} U_{R^{*}}$ containing ${ }_{S} C_{R^{*}}$, such that $E\left({ }_{s} C\right)={ }_{s} U$.

An Example (of the Bad Case) with Hereditary $R^{*}$. As in [12, Example A], let $R$ (respectively, $S$ ) be the algebra given by the quiver

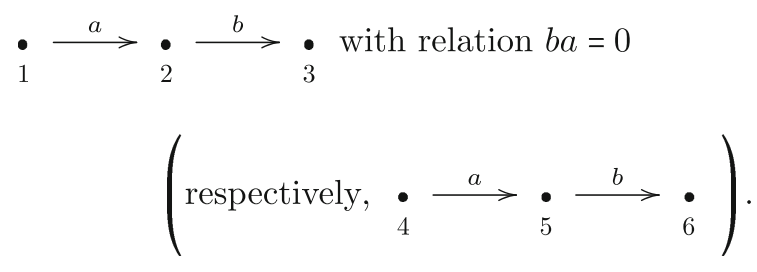

Let ${ }_{S} C_{R}$ be the cotilting bimodule such that ${ }_{S} C=6 \oplus \begin{aligned} & 4 \\ & 5\end{aligned} \oplus 4$ and $C_{R}={ }_{6}^{3} \oplus 2 \oplus \begin{aligned} & 2 \\ & 2\end{aligned} \oplus$. Next, let $R^{*}$ be the hereditary algebra given by the Dynkin diagram

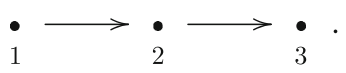

Finally, let $F: R^{*} \longrightarrow R$ be the obvious ring epimorphism. Then $\operatorname{dim} \operatorname{ker} F=1$ and $C$, regarded as a $S-R^{*}$ bimodule, is contained in the $S-R^{*}$ bimodule $U$, satisfying (i) and (ii), described in Picture 6.

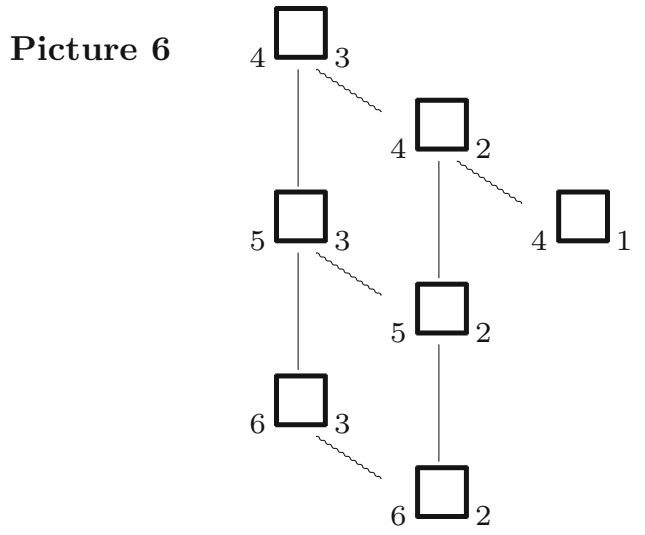

An Example (of the Bad Case) with Nonhereditary $\mathbf{R}^{*}$. As in [12, Example C], let $R$ (respectively, $S$ ) be the algebra given by the quiver $\stackrel{\vec{b}}{\stackrel{a}{\rightleftarrows}}$. with relation $a b=0$ (respectively, $\stackrel{c}{\stackrel{c}{\rightleftarrows}}$. with relation $c d=0$ ). Next, let ${ }_{s} C_{R}$ be the 
cotilting bimodule such that ${ }_{S} C=\begin{aligned} & 3 \\ & 4\end{aligned}$ B 3 and $C_{R}=2 \oplus 1$. Next, let $R^{*}$ denote the algebra given by the quiver $\overbrace{1} \stackrel{a}{\longrightarrow}$ e with relation $a b a=0$. Finally, let $F$ : $R^{*} \longrightarrow R$ be the obvious ring epimorphism. Then $\operatorname{dim} \operatorname{Ker} F=2$ and $C$ regarded as an $S-R^{*}$ bimodule is contained in the $S-R^{*}$ bimodule $U$, satisfying (i) and (ii), described in Picture 7.

\section{Picture 7}

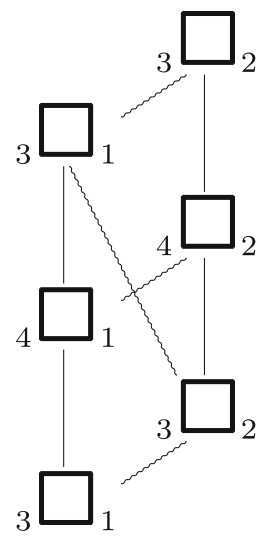

Let us wrap this section up with two open problems on bimodules.

Problem 1. Are the conditions of the "Remarks on the Action of Primitive Idempotents and Nilpotent Elements in the Good Case" section satisfied by any bimodule as in the good case described in the section preamble?

Problem 2. Are conditions (i) and (ii) of the section entitled "Remarks on the Action of New Concealed Rings in the Bad Case" satisfied by any bimodule as in the bad case described in the section preamble?

\section{Do Infinite-Dimensional Modules Need No Topological Tools?}

Another reason for surprise at the presence of the kind of topology described in the section entitled "Do Finite-Dimensional Bimodules Have a Topology?" (see the remark after Picture 5) is the absence of topological arguments in the proof of a result concerning dualities induced by cotilting bimodules of infinite dimension. Before we discuss this, we note that a left $S$-module (respectively, a right $R$-module) $M$ is reflexive with respect to the bimodule ${ }_{S} U_{R}$ (or just U-reflexive or reflexive, for short) if $M$ is canonically isomorphic to its double dual with respect to $U$, that is, to the module $\Delta(\Delta(M))$, where $\Delta$ denotes both the contravariant functors $\operatorname{Hom}_{?}\left(-{ }_{S} U_{R}\right)$ for $?=R, S$, and the group $\Delta(X)$ is equipped with its module structure (see [1, Proposition 4.4] or [23, Propositions 3.4 and 3.5]) for any left $S$-module and any right $R$-module $X$.

Obvious and Nonobvious Reflexive Modules. Even in special cases, for instance, given a faithfully balanced bimodule $U$, there is a big gap between the well-known indecomposable reflexive modules, which are either projective or summands of $U$ [1, Propositions 20.13 and 20.14 and Corollary 20.16] and the rest of the world, that is, the nonobvious indecomposable reflexive modules.

The cotilting bimodule described in the section entitled "Toy Example of a Finite-Dimensional Cotilting Bimodule" admits four indecomposable reflexive left (respectively, right) modules. Moreover, comparing Auslander-Reiten quivers (and looking at Picture 2), we see that all of them are obvious reflexive modules and the duality $\Delta$ acts as follows:

$$
\begin{aligned}
& 4 \\
& 5
\end{aligned} \frac{2}{6}, \begin{aligned}
& 5 \\
& 6
\end{aligned}{ }_{23}^{1}, 5 \mapsto 3,6 \mapsto \begin{aligned}
& 1 \\
& 2
\end{aligned} .
$$

A similar situation holds for the cotilting module described in the "An Example (of the Bad Case) with Hereditary R*" section (respectively, the "An Example (of the Bad Case) with Nonhereditary $\mathrm{R}^{* \text { " }}$ section, where the cotilting duality $\Delta$ acts as follows:

$$
\begin{aligned}
& 6 \mapsto \begin{array}{l}
3 \\
2
\end{array}, \begin{array}{l}
4 \\
5 \\
6
\end{array} \mapsto \begin{array}{l}
2 \\
1
\end{array}, 4 \mapsto 1, \begin{array}{l}
5 \\
6
\end{array} \mapsto 2 \\
& \text { (respectively, } \left.\begin{array}{rrr}
3 & & 1 \\
3 & & 2 \\
& 1
\end{array}, 3 \mapsto \begin{array}{l}
2 \\
1
\end{array}, \begin{array}{l}
4 \\
3
\end{array} \mapsto 1\right) \text {. }
\end{aligned}
$$

On the other hand, Happel-Ringel's cotilting (and tilting) bimodule $T$ described in the "A Letter from Japan and My First Homework on Tilting Theory" section easily gives us an example of a cotilting bimodule admitting nonobvious reflexive modules. Indeed, by comparing Auslander-Reiten quivers (and by looking at Picture 1), it is easy to see that $T$ admits fourteen indecomposable reflexive left and right modules, respectively. But ${ }_{6}^{5}$ is the unique indecomposable projective summand of the left $A$-module $T$. Therefore $T$ admits eleven obvious and three nonobvious indecomposable reflexive left and right modules, respectively. We already described (at the end of the "A Letter from Japan and My First Homework on Tilting Theory" section) how the cotilting duality $\Delta$ acts on the indecomposable summands of ${ }_{A} T$. Now $\Delta$ acts on the remaining obvious left $A$-modules, that is, the five indecomposable projective modules that are not summands of ${ }_{A} T$, via:

$$
\begin{aligned}
& \begin{array}{l}
1 \\
2 \\
6
\end{array} \mapsto \begin{array}{l}
d \\
e
\end{array}, \quad \begin{array}{l}
2 \\
6
\end{array} \mapsto \begin{array}{c}
b d, \\
e
\end{array}, \begin{array}{l}
3 \\
4 \\
6
\end{array} \mapsto \begin{array}{l}
d \\
f
\end{array},
\end{aligned}
$$

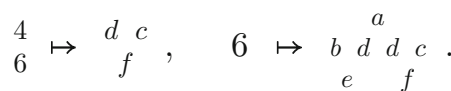

Finally, $\Delta$ acts on the three indecomposable nonobvious reflexive left $A$-modules via:

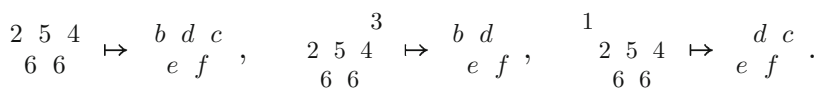

Several important results about nonobvious modules can be obtained by means of rather technical topological tools (see [8, Sections 2 and 3], [7, 9, 10], and the papers cited in [8]). That's why it was a pleasant surprise to see that a 
discrete bimodule was enough to answer Colpi's question: "Are reflexive modules closed under submodules?" His additional hint was "You cannot use finite-dimensional algebras and modules!" (See [9, Theorem 1] for the nice behavior of submodules of reflexive modules over Artin algebras.)

Proposition [11, Lemma 2.4 and Theorem 2.5 (ii)]. Reflexive modules with respect to a cotilting bimodule are not necessarily closed under submodules. Moreover, even the well-known reflexive modules with respect to a faithfully balanced bimodule ${ }_{S} U_{R}$, that is, the indecomposable summands of both the left (respectively, right) regular module $S$ (respectively, $R$ ) and of the module $U$ are not necessarily closed under submodules.

The next example-more precisely, the next pictureshows that the above result has a purely combinatorial motivation coming from basic linear algebra. Indeed, the result follows from the same reason that an infinite-dimensional $K$-vector space cannot be isomorphic to its double dual, and so it cannot be reflexive with respect to the regular bimodule ${ }_{K} K_{K}$. (See [8, Proposition 1.8] for a general result on direct sums of infinitely many nonzero reflexive modules with respect to a cotilting bimodule.)

Toy Example of a Finite-Dimensional Cotilting Bimodule as in the previous Proposition [11, Lemma 2.4 and Theorem 2.5 (ii)] . With terminology suggested by [22], assume $R=S$ is the "generalized" Kronecker algebra given by the quiver

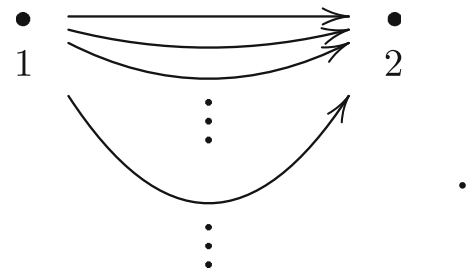

Then the indecomposable projective nonsimple left (respectively, right) module is a reflexive module with respect to the cotilting bimodule ${ }_{R} R_{R}$. However, its maximal submodule, that is, the Jacobson radical of $R$, generated by the infinitely many arrows from 1 to 2 , is not reflexive with respect to $R$ :

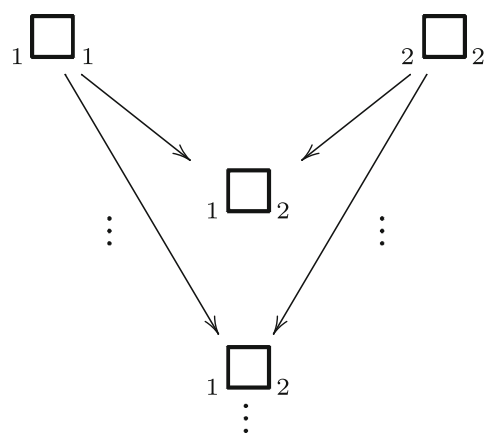

\section{Final Observations}

In the world of tilting and cotilting, indecomposable projective and injective modules play a big role, as well as indecomposable projective-injective modules. This justifies the thinking that tilting objects exist in nature and are not artificial. The examples considered in this article (concerning tilting modules of projective dimension at most one and cotilting modules of injective dimension at most one) also suggest that classical tilting and cotilting modules are complicated enough to solve interesting questions, as the question on reflexive modules presented previously.

I should confess that I always try to use these modules (and in particular the pictures describing them) to visualize more-or-less mysterious equivalences and dualities. However I always prefer to use the long and combinatorial definitions of tilting and cotilting-type objects, that is, the definitions where classes of modules do not appear directly. I view the existence of completely different definitions as a proof of the depth of these objects. Indeed they have both combinatorial and functorial properties and it is possible to define them by means of either set of properties separately.

Through the years, tilting objects became more and more abstract and were defined over Grothendieck categories and triangulated categories. However I believe that the original notion of a tilting module is still very significant. I finally recall that a word coming from physics-reflection-appeared in the title of the first paper [5] by Brenner and Butler on tilting theory. Sheila Brenner told me they extensively discussed options for a suggestive name; I believe they succeeded in finding one.

\section{ACKNOWLEDGMENTS}

I would like to thank colleagues in Milan, Ferrara, and Sherbrooke for opportunities to present this work to engaged audiences, and the referees and editors for helpful suggestions.

Department of Mathematics

University of Milano

Via Saldini 50

20133 Milano

Italy

e-mail: gabriella.deste@unimi.it

\section{OPEN ACCESS}

This article is distributed under the terms of the Creative Commons Attribution 4.0 International License (http:// creativecommons.org/licenses/by/4.0/), which permits unrestricted use, distribution, and reproduction in any medium, provided you give appropriate credit to the ori ginal author(s) and the source, provide a link to the Creative Commons license, and indicate if changes were made.

\section{REFERENCES}

[1] F. W. Anderson and K. R. Fuller, Rings and categories of modules, GTM 13, Springer-Verlag (1992). 
[2] L. Angeleri-Hügel, D. Happel, and H. Krause, Handbook of Tilting Theory, London Mathematical Society Lecture Note Series 332, Cambridge University Press (2007).

[3] M. Auslander, I. Reiten, and S. O. Smalø, Representation theory of artin algebras, Cambridge University Press, Cambridge (1995).

[4] S. Bazzoni, A characterization of $n$-cotilting and $n$-tilting modules, J. Algebra 273 (2004), 359-372.

[5] S. Brenner and M. C. R. Butler, Generalizations of the Bernstein-Gelfand-Ponomarev reflection functors, Representation Theory II (Ottawa, 1979), V. Dlab and P. Gabriel (eds.), Lec. Notes in Math., 832, Springer, Berlin (1980), 103-169.

[6] R. Colpi, Tilting modules and $*$-modules, Comm. Algebra 21(4) (1993), 1095-1102.

[7] R. Colpi, Cotilting bimodules and their dualities, Proc. Euroconf. Murcia '98, LNPAM 210, M. Dekker (2000), 81-93.

[8] R. Colpi, Dualities induced by cotilting bimodules, LNPAM 236 , M. Dekker (2004), 89-102.

[9] R. R. Colby, R. Colpi, and K. R. Fuller, A note on generalized modules and generalized Morita dualities, LNPAM 236, M. Dekker (2004), 85-88.

[10] R. Colpi and K. R. Fuller, Cotilting modules and bimodules, Pacific J. Math. 192 (2000), 275-291.

[11] G. D'Este, Reflexive modules are not closed under submodules, Representations of algebras (Sao Paulo), LNPAM 224, M. Dekker (2002), 53-64.

[12] G. D'Este, Symmetries and asymmetries for cotilting bimodules, LNPAM 236, M. Dekker (2004), 103-118.

[13] G. D'Este, Comparing small orthogonal classes, Generalized Lie Theory in Mathematics, Physics and Beyond, S. Silvestrov, E. Paal, and V. Abramov (eds.), Springer (2009), 119-128.

[14] G. D'Este, Orthogonal classes and their more or less concealed subclasses, IJPAM 44(4) (2008), 627-643.

[15] G. D'Este, On partial tilting modules and bounded complexes, Israel Journal of Math. 16 (2008), 17-38.

[16] G. D'Este, On partial tilting modules and right bounded complexes, Ann. Univ. Ferrara 57 (2011), 245-260.

[17] G. D'Este, Looking for right bounded complexes, Groups and Model Theory: A Conference in Honor of Rüdiger Göbel's 70th Birthday, CONM AMS 576 (2012), 41-56.

[18] V. Dlab, Representation of valued graphs: (notes du cours à la 18 session du Séminaire de Math. Sup. au Dép. de Math. et de Statist. de I'Univ. de Montréal, 11 Juin-6 Julliet 1979), Presses de I'Univ. de Montréal (1980).
[19] V. Dlab and C. M. Ringel, Indecomposable representation of graphs and algebras, Mem. Amer. Math. Soc. 173 (1976).

[20] Information list for representation theory of finite dimensional algebras, http://www.math.uni-bielefeld.de/ follist.

[21] D. Happel and C. M. Ringel, Tilted algebras, Trans. Amer. Math. Soc. 274 (1982), 399-443.

[22] D. Happel and L. Unger, A family of infinite-dimensional non selfextending bricks for wild hereditary algebras, CMS Conference Proceedings 114 (1991), 181-189.

[23] N. Jacobson, Basic Algebra II, Freeman and Co., San Francisco (1980).

[24] O. Kerner and J. Trlifaj, Constructing tilting modules, Trans. Amer. Math. Soc. 360 (2008), 1907-1925.

[25] F. Mantese, Hereditary cotilting modules, J. Algebra 238 (2001), 662-678 .

[26] F. Mantese, Complements of projective almost complete tilting modules, Communications in Algebra 33(4) (2005), 2921-2940.

[27] C. Menini, Orsatti's contribution to Module Theory, LNPAM 201 (1998), 9-18.

[28] C. Menini and A. Orsatti, Representable equivalences between categories of modules and applications, Rend. Sem. Mat. Univ. Padova 82 (1989), 203-231.

[29] C. M. Ringel, Unzerlegbare Darstellungen endlich-dimensionaler Algebren., Jber. d. Dt. Math. Verein. 85 (1983), 86-105.

[30] C. M. Ringel, Infinite length modules. Some examples as introduction, in "Infinite Length Modules," H. Krause and C. M. Ringel, Trends in Mathematics, Birkhäuser Verlag (2000), 1-73.

[31] C. M. Ringel, Combinatorial Representation Theory: History and Future, Representations of Algebras, Vol. I, D. Happel and Y. B. Zhang (eds.), BNU Press (2002), 122-144.

[32] C. M. Ringel, Tame algebras and integral quadratic forms, Springer LMN 1099 (1984).

[33] C. M. Ringel, The art of losing modules (March 16, 2007), Fully documented lectures, section "Lectures" in Ringel's homepage. www.math.uni-bielefeld.de/ ringel/lectures.html.

[34] J. Rickard, Morita theory for derived categories, J. London Math. Soc. 39(2) (1989), 436-456.

[35] M. Schaps and E. Zakay-Illouz, Combinatorial partial tilting complexes for the Brauer star algebras, Lecture Notes in Pure and Appl. Math., volume 224, Marcel Dekker (2002), 187-207.

[36] P. Vamos, The Holy Grail of algebra: seeking complete sets of invariants, Mathematics and Its Applications 343, Kluwer (1995), 475-490. 